habit which is well known to be variable. The actual somatic expression of the variation is defined by imperfectly understood laws of growth and functioning, which are themselves determined by the hereditary nature of the developing material. But in divergent as in normal expressions of the developing material, much may depend on the liberating stimuli which individual nurture affords.

The bulk of the book (pp. 97-143) is occupied with the reports of the individual cases. "Much ery and little wool", it may be said, but we cannot agree. We think the author has done well to make the most of a picturesque human variation; but we wish that his disciplined anthropological experience had had 3000 , not 300 , cases to work with. We also wish that he had been able to say a little more in regard to the very interesting similar phenomena in children, namely, those activities that look like recapitulations or rehabilitations of ancestral features. Thus many children have a strongly defined predisposition to climb trees, which may be subtly linked back to man's arboreal apprenticeship, of which his body retains many marks, as has been indicated in detail by $R$. Anthony and by F. Wood Jones. Also very familiar is the prehensility of the toes in many young children, who will lift a pencil or the like with their toes to their hands or to their mouth. These are but examples of what is well known. There is still much to be discovered in regard to the way in which the past lives on in the present.

\section{Adsorption Data}

The Sorption of Gases and Vapours by Solids. By Prof. J. W. McBain. (Twentieth-Century Chemistry, 4.) Pp. xii +577. (London : George Routledge and Sons, Ltd., 1931.) 25s. net.

DECENTLY it has been stated that on the 1 average a paper on adsorption appears every day in the year. Prof. J. W. McBain's volume is a masterpiece of collection, codification, and classification of such data. It is divided into three parts, the introductory section, the experimental data, and the hypotheses and theories of sorption.

The second section (pp. 33-426), which is certainly the most complete compilation on the subject ever published, includes not only a very comprehensive survey of the literature on sorption by charcoal and other porous rigid systems, but also an account of the work carried out on metals, cellulose, and nonrigid gels. The section is not only singularly com- plete, but also extremely interesting to read, as the author has from time to time inserted personal com. ments and criticism. It is, on the other hand, not so satisfactory if regarded as an introduction to the third section, which includes the theories of adsorption. The reviewer has found it very difficult 'to see the wood for the trees'. To take only two examples. We know with some degree of certainty that practically all cases of adsorption are accompanied by a penetration of the gas or vapour into the interior of the solid by processes which may include space-lattice diffusion, persorption through molecular sieves, intergranular diffusion and capillary penetration, and it is indeed to Prof. McBain himself that we are largely indebted for a proper appreciation of the importance of this phenomenon. Again, from the work of Dewar on the oxygen charcoal system, of Ostwald and Langmuir on the system $\mathrm{CaO}-\mathrm{CO}_{2}$, it was clear that at least two states of adsorption existed. The work of Benton and of Nikitin has revealed another state, the existence of which has been confirmed by the more recent work of H. S. Taylor and of Garner. These three types of adsorption, sometimes termed Van der Waals' adsorption, activated adsorption, and chemi-adsorption respectively, all exhibit their own peculiar characteristics, and examples of all three types can indeed occur in certain systems when taken over a sufficiently wide range of temperature. Whilst the actual facts are presented to the reader, a dissection of the data by Prof. McBain, or even an arrangement of the data in such a manner so as to exemplify those points, would provide a reader with a valuable introduction to the theoretical section.

In the theoretical section, the author subjects the hypothesis of adsorption in multimolecular layers to a somewhat detailed analysis, concluding that Langmuir's hypothesis of monomolecular adsorp tion is adequate to fit all the data which have been established with sufficient degree of certainty The discussion on the more recent developments of Langmuir's original hypothesis, such as surface mobility and the necessity for energies of activation after 'primary' adsorption, is particularly interesting. More might have been included in the section on active patches and promoters, on the importance of lattice spacing as distinct from isolated atoms or configuration as factors in reaction at or with a surface.

The volume is full of most interesting information clearly presented, and can certainly be recom. mended to all those who are interested in the phenomena of sorption.

Eric K. Rideal. 